\title{
Automated Theorem Proving in the Homogeneous Model with Clifford Bracket Algebra
}

\author{
Hongbo $\mathrm{Li}^{1)}$
}

A Clifford algebra has three major multiplications: inner product, outer product and geometric product. Accordingly, the same Clifford algebra has three versions: Clifford vector algebra, which features on inner products and outer products of multivectors; Clifford bracket algebra, which features on pseudoscalars and inner products of vectors; Clifford geometric algebra, which features on geometric products of vectors and multivectors.

Since 1993, there have been various applications of Clifford vector algebras in automated geometric theorem proving [9], [10], [11], [12], [13], [14], [3], [15], [16], [17], [18]. Some famous theorems which are difficult to prove mechanically by other methods, either algebraic or logical ones, have been proved successfully with Clifford vector algebra methods. 2D Clifford geometric algebra is just the algebra of complex numbers, its applications in automated theorem proving can be found in [2], [9], etc. For projective geometry, bracket algebra is very important for invariant geometric computing [24], [25], [26],[27] and automated theorem proving [21], [22]. For Euclidean geometry, complex bracket algebra [21], distance algebra [4] and the method of areas and Pythagorean distances [2], have been efficiently used in theorem proving.

From the brief summary, we notice that Clifford bracket algebra, whose foundation is a set of generalized Grassmann-Plücker relations, has not been systematically applied to theorem proving in Euclidean geometry. For Euclidean geometry, a very useful Clifford algebraic model is the homogeneous model [5], [20], [19]. The Clifford bracket algebra in the homogeneous model is what we choose for invariant and automated theorem proving in Euclidean geometry.

This chapter presents some of our newest results in applying this Clifford bracket algebra in 2D Euclidean geometric theorem proving. The highlight is that some tremendously difficult geometric computing tasks can be finished with the Clifford bracket algebra, but not by any other pure algebraic methods when running on currently available computer systems.

The following is a typical example - the five-circle theorem: Let $A, B, C, D, E$ be five generic points in the plane. Let

$$
\begin{aligned}
& 1=A E \cap B C, \quad 2=A B \cap C D, \quad 3=B C \cap D E, \\
& 4=A E \cap C D, \quad 5=A B \cap D E .
\end{aligned}
$$

Let $A B C$ be the circle passing through $A, B, C$, and let $A B C \cap A D E$ be the point other than $A$ at the intersection of circles $A B C$ and $A D E$ if they intersect, or $A$ if they are tangent to

Academy of Mathematics and System Sciences, Chinese Academy of Sciences, Beijing 100080, China 
each other. Let

$$
\begin{array}{ll}
a=A B 1 \cap A E 5, & b=A B 1 \cap B C 2, \quad c=B C 2 \cap C D 3, \\
d=C D 3 \cap D E 4, & e=D E 4 \cap A E 5 .
\end{array}
$$

Then $a, b, c, d, e$ are on the same circle, called cyclic.

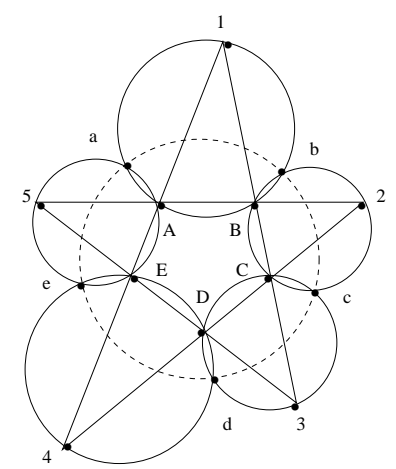

Fig. 1. The five-circle Theorem.

Previous proofs of this theorem are either classical Euclidean, or based on dynamic database and forward chaining (e.g., the full-angles method in [2]). No algebraic proof has ever been found, although theoretically, an algebraic proof is "very easy" to establish: by symmetry we only need to prove that $a, b, c, d$ are cyclic; since the coordinates of $A B C \cap A D E$ can be explicitly expressed by those of $A, B, C, D, E$, we have

$$
\begin{array}{ll}
a=a(A, B, C, D, E), & b=b(A, B, C, D, E), \\
c=c(A, B, C, D, E), & d=d(A, B, C, D, E),
\end{array}
$$

so an algebraic proof is reduced to verifying the Plücker equality:

$$
\begin{gathered}
|a b||c d| \pm|a c||b d| \pm|a d||b c|=0, \text { where }|a b| \text { denotes the distance; } \\
\text { i.e., }\left|\begin{array}{cccc}
0 & |a b| & |a c| & |a d| \\
|a b| & 0 & |b c| & |b d| \\
|a c| & |b c| & 0 & |c d| \\
|a d| & |b d| & |c d| & 0
\end{array}\right|=0 .
\end{gathered}
$$

This is a task of pure algebraic computing.

\section{Clifford bracket algebra}

Let $\mathcal{V}^{n}$ be a real nondegenerate inner product space of dimension $n, \mathcal{G}_{n}$ be the Clifford algebra generated by $\mathcal{V}^{n}$. The bracket of a multivector $A$ is defined by $[A]=\left(\langle A\rangle_{n}\right)^{*}$.

Let $\mathbf{a}_{1}, \ldots, \mathbf{a}_{m}$ be indeterminates in $\mathcal{V}^{n}, m \geq n$. The Clifford bracket algebra generated by the $\mathbf{a}$ 's is the quotient of the polynomial ring generated by all nonzero brackets $\left[\mathbf{a}_{i_{1}} \mathbf{a}_{i_{2}} \ldots \mathbf{a}_{i_{n}}\right]$ and all inner products of vectors $\mathbf{a}_{i} \cdot \mathbf{a}_{j}$, by the two-sided ideal generated by the following generalized Grassmann-Plücker relations: 
Group 1. $\sum_{k=1}^{n+1}(-1)^{k+1} \mathbf{a}_{j} \cdot \mathbf{a}_{i_{k}}\left[\mathbf{a}_{i_{1}} \ldots \check{\mathbf{a}}_{i_{k}} \ldots \mathbf{a}_{i_{n+1}}\right]=0$.

Group 2. $\left[\mathbf{a}_{i_{1}} \ldots \mathbf{a}_{i_{n}}\right]\left[\mathbf{a}_{j_{1}} \ldots \mathbf{a}_{j_{n}}\right]=(-1)^{\frac{n(n-1)}{2}} \operatorname{det}\left(\mathbf{a}_{i_{k}} \cdot \mathbf{a}_{j_{l}}\right)_{k, l=1 . . n}$.

The following relations are generated by the above ones:

Group 3. (Grassmann-Plücker relations)

$\sum_{k=1}^{n+1}(-1)^{k+1}\left[\mathbf{a}_{i_{1}} \ldots \check{\mathbf{a}}_{i_{k}} \ldots \mathbf{a}_{i_{n+1}}\right]\left[\mathbf{a}_{i_{k}} \mathbf{a}_{j_{1}} \ldots \mathbf{a}_{j_{n-1}}\right]=0$.

Group 4. (Van der Waerden relations) For any $1 \leq r \leq n$,

$\sum_{\sigma^{r}} \operatorname{sign}\left(\sigma^{r}\right)\left[\mathbf{a}_{i_{1}} \ldots \mathbf{a}_{i_{n-r}} \mathbf{a}_{\sigma_{1}^{r}} \ldots \mathbf{a}_{\sigma_{r}^{r}}\right]\left[\mathbf{a}_{\sigma_{r+1}^{r}} \ldots \mathbf{a}_{\sigma_{n+1}^{r}} \mathbf{a}_{j_{1}} \ldots \mathbf{a}_{j_{r-1}}\right]=0$,

where $\sigma^{r}$ is a permutation of $1, \ldots, n+1$ such that $\sigma^{r}(1)=\sigma_{1}^{r}<\cdots<\sigma_{r}^{r}$ and $\sigma_{r+1}^{r}<\cdots<$ $\sigma_{n+1}^{r}$.

Group 5. $\operatorname{det}\left(\mathbf{a}_{i_{k}} \cdot \mathbf{a}_{j_{l}}\right)_{k, l=1 . . n+1}=0$.

We will use only the Clifford bracket algebra for the homogeneous model below. Let $\mathcal{R}^{n-1,1}$ be $n \mathrm{D}$ Minkowski space, $\mathcal{N}=\left\{\mathbf{a} \in \mathcal{R}^{n-1,1} \mid \mathbf{a}^{2}=0, \mathbf{a} \neq 0\right\}$ be the set of null vectors. Fixing $\mathbf{f} \in \mathcal{N}$, the set

$$
\mathcal{N}_{\mathbf{f}}=\{\mathbf{a} \in \mathcal{N} \mid \mathbf{a} \cdot \mathbf{f} \neq 0\}
$$

is projectively homeomorphic to $\mathcal{R}^{n-2}$. So

$$
\mathcal{N}_{\mathbf{f}}^{1}=\{\mathbf{a} \in \mathcal{N} \mid \mathbf{a} \cdot \mathbf{f}=-1\}
$$

is homeomorphic to $\mathcal{R}^{n-2}$.

When another vector $\mathbf{f}_{0} \in \mathcal{N}_{\mathbf{f}}^{1}$ is also fixed, then $\left\langle\mathbf{f}, \mathbf{f}_{0}\right\rangle^{\perp}$ is an $n \mathrm{D}$ Euclidean space. The mapping

$$
\begin{aligned}
\left\langle\mathbf{f}, \mathbf{f}_{0}\right\rangle^{\perp} & \longrightarrow \mathcal{N}_{\mathbf{f}}^{1} \\
a & \mapsto \quad \mathbf{a}=\mathbf{f}_{0}+a+\frac{a^{2}}{2} \mathbf{f}
\end{aligned}
$$

satisfies

- $\mathbf{a} \cdot \mathbf{b}=-\frac{|a b|^{2}}{2}$.

- $[\mathbf{f a b c}]=-2 S_{a b c}$, where $S_{a b c}$ is the signed area of triangle $a b c$. In particular, points $a, b, c$ are collinear if and only if $[\mathbf{f a b c}]=0$.

- Let $o$ and $\rho$ be respectively the center and radius of circle $a b c$, then [abcd] $=\left(|o d|^{2}-\right.$ $\left.\rho^{2}\right) S_{a b c}$. In particular, points $a, b, c, d$ are on the same circle if and only if [abcd] $=0$.

The homogeneous model of Euclidean geometry has three versions:

Projective version: $\mathcal{N}_{\mathbf{f}}$. For collinear and co-spherical relations only.

Affine version: $\mathcal{N}_{\mathbf{f}}^{1}$. For origin-free computing.

Euclidean version: $\left(\mathcal{N}_{\mathbf{f}}^{1}, \mathbf{f}_{0}\right)$. Full version.

Let $\mathbf{a}_{1}, \ldots, \mathbf{a}_{m}$ be null vectors in $\mathcal{R}^{n-1,1}, m \geq n$. The corresponding Clifford bracket algebra in the homogeneous model is the quotient of the polynomial ring generated by all 
nonzero brackets $\left[\mathbf{a}_{i_{1}} \mathbf{a}_{i_{2}} \ldots \mathbf{a}_{i_{n}}\right]$ and all inner products of vectors $\mathbf{a}_{i} \cdot \mathbf{a}_{j}$ for $i<j$, by the two-sided ideal generated by the generalized Grassmann-Plücker relations and the squares of the a's.

\section{Some bracket expressions}

There are several important bracket expressions that occur in geometric constructions. Below we present two of them. From now on, $n=4$.

By a generalized circle we mean a circle or straight line in the plane, by a generalized point we mean a point or the point at infinity represented by $\mathbf{f}$. Let $b$ be the other generalized point other than $a$ at the intersection of generalized circles $a a_{1} a_{2}$ and $a a_{3} a_{4}$ if they intersect, or $a$ if they are tangent to each other (two parallel lines are tangent at the point at infinity). Then

$$
\begin{aligned}
& \mathbf{b}=\mathbf{a}_{3} \cdot \mathbf{a}_{4}\left[\mathbf{a} \mathbf{a}_{1} \mathbf{a}_{2} \mathbf{a}_{3}\right]\left[\mathbf{a} \mathbf{a}_{1} \mathbf{a}_{2} \mathbf{a}_{4}\right] \mathbf{a}-\left[\mathbf{a} \mathbf{a}_{1} \mathbf{a}_{2} \mathbf{a}_{4}\right]\left[\mathbf{a}_{1} \mathbf{a}_{2} ; \mathbf{a}_{3} \mathbf{a}_{4}\right]_{\mathbf{a}} \mathbf{a}_{3} \\
& +\left[\mathbf{a} \mathbf{a}_{1} \mathbf{a}_{2} \mathbf{a}_{3}\right]\left[\mathbf{a}_{1} \mathbf{a}_{2} ; \mathbf{a}_{3} \mathbf{a}_{4}\right]_{\mathbf{a}} \mathbf{a}_{4} \\
& =\mathbf{a}_{1} \cdot \mathbf{a}_{2}\left[\mathbf{a} \mathbf{a}_{1} \mathbf{a}_{3} \mathbf{a}_{4}\right]\left[\mathbf{a} \mathbf{a}_{2} \mathbf{a}_{3} \mathbf{a}_{4}\right] \mathbf{a}+\left[\mathbf{a} \mathbf{a}_{2} \mathbf{a}_{3} \mathbf{a}_{4}\right]\left[\mathbf{a}_{1} \mathbf{a}_{2} ; \mathbf{a}_{3} \mathbf{a}_{4}\right]_{\mathbf{a}} \mathbf{a}_{1} \\
& -\left[\mathbf{a a}_{2} \mathbf{a}_{3} \mathbf{a}_{4}\right]\left[\mathbf{a}_{1} \mathbf{a}_{2} ; \mathbf{a}_{3} \mathbf{a}_{4}\right]_{\mathbf{a}} \mathbf{a}_{2}
\end{aligned}
$$

where

$$
\begin{aligned}
{\left[\mathbf{a}_{1} \mathbf{a}_{2} ; \mathbf{a}_{3} \mathbf{a}_{4}\right]_{\mathbf{a}} } & =\left[\mathbf{a} \mathbf{a}_{2} \mathbf{a}_{3} \mathbf{a}_{4}\right] \mathbf{a} \cdot \mathbf{a}_{1}-\left[\mathbf{a} \mathbf{a}_{1} \mathbf{a}_{3} \mathbf{a}_{4}\right] \mathbf{a} \cdot \mathbf{a}_{2} \\
& =\left[\mathbf{a} \mathbf{a}_{1} \mathbf{a}_{2} \mathbf{a}_{3}\right] \mathbf{a} \cdot \mathbf{a}_{4}-\left[\mathbf{a} \mathbf{a}_{1} \mathbf{a}_{2} \mathbf{a}_{4}\right] \mathbf{a} \cdot \mathbf{a}_{3}
\end{aligned}
$$

is multilinear with respect to $\mathbf{a}_{1}, \mathbf{a}_{2}, \mathbf{a}_{3}, \mathbf{a}_{4}$ and satisfies

$$
\left[\mathbf{a}_{1} \mathbf{a}_{2} ; \mathbf{a}_{3} \mathbf{a}_{4}\right]_{\mathbf{a}}=-\left[\mathbf{a}_{2} \mathbf{a}_{1} ; \mathbf{a}_{3} \mathbf{a}_{4}\right]_{\mathbf{a}}=-\left[\mathbf{a}_{1} \mathbf{a}_{2} ; \mathbf{a}_{4} \mathbf{a}_{3}\right]_{\mathbf{a}}=-\left[\mathbf{a}_{3} \mathbf{a}_{4} ; \mathbf{a}_{1} \mathbf{a}_{2}\right]_{\mathbf{a}}
$$

1. Let the center and radius of circle $a a_{1} a_{2}$ be $o_{12}, \rho_{12}$, and let the center and radius of circle $a a_{3} a_{4}$ be $o_{34}, \rho_{34}$. Let the signed distance from point $c$ to ray $a b$ be $d(c, a b)$. Then

$$
\begin{aligned}
{\left[\mathbf{a}_{1} \mathbf{a}_{2} ; \mathbf{a}_{3} \mathbf{a}_{4}\right]_{\mathbf{a}} } & =-4 \rho_{34} d\left(o_{12}, o_{34} a\right) S_{a a_{1} a_{2}} S_{a a_{3} a_{4}} \\
& =4 \rho_{12} d\left(o_{34}, o_{12} a\right) S_{a a_{1} a_{2}} S_{a a_{3} a_{4}} .
\end{aligned}
$$

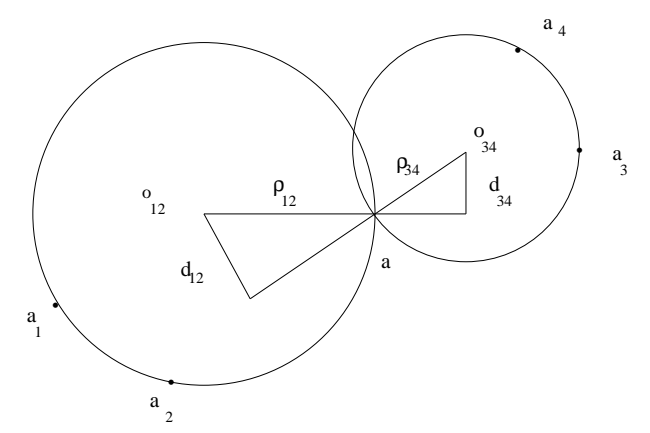

Fig. 2. Interpretation of $\left[\mathbf{a}_{1} \mathbf{a}_{2} ; \mathbf{a}_{3} \mathbf{a}_{4}\right]_{\mathbf{a}}$.

$\left[\mathbf{a}_{1} \mathbf{a}_{2} ; \mathbf{a}_{3} \mathbf{a}_{4}\right]_{\mathbf{a}}=0$ if and only if circles $a a_{1} a_{2}, a a_{3} a_{4}$ are either tangent to each other or identical. 
2. If $\mathbf{a}=\mathbf{f}$, then $b=a_{1} a_{2} \cap a_{3} a_{4}$ if the two lines intersect, or $\mathbf{b}=\mathbf{f}$ if they are parallel to each other. We have

$$
\left[\mathbf{a}_{1} \mathbf{a}_{2} ; \mathbf{a}_{3} \mathbf{a}_{4}\right]=\left[\mathbf{a}_{1} \mathbf{a}_{2} ; \mathbf{a}_{3} \mathbf{a}_{4}\right]_{\mathbf{f}}=-2 S_{a_{1} a_{3} a_{2} a_{4}}
$$

where $S_{a_{1} a_{3} a_{2} a_{4}}$ is the signed area of quadrilateral $a_{1} a_{3} a_{2} a_{4}$.

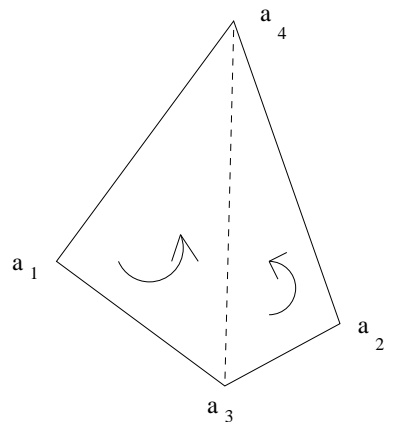

Fig. 3. Interpretation of $\left[\mathbf{a}_{1} \mathbf{a}_{2} ; \mathbf{a}_{3} \mathbf{a}_{4}\right]$.

In the general case, $-\left[\mathbf{a}_{1} \mathbf{a}_{2} ; \mathbf{a}_{3} \mathbf{a}_{4}\right]$ is the signed area of the parallelogram spanned by vectors $a_{1} a_{2}$ and $a_{3} a_{4}$, and $\left[\mathbf{a}_{1} \mathbf{a}_{2} ; \mathbf{a}_{3} \mathbf{a}_{4}\right]=0$ if and only if $a_{1} a_{2} \| a_{3} a_{4}$.

Let $a_{1} a_{2} a_{3} a_{5} a_{4}$ be a pentagon, then its signed area is $S_{a_{1} a_{2} a_{3} a_{5} a_{4}}=-\left[\mathbf{a}_{1} ; \mathbf{a}_{2} \mathbf{a}_{3} ; \mathbf{a}_{4} \mathbf{a}_{5}\right] / 2$, where

$$
\begin{aligned}
& {\left[\mathbf{a}_{1} ; \mathbf{a}_{2} \mathbf{a}_{3} ; \mathbf{a}_{4} \mathbf{a}_{5}\right]=\mathbf{f} \cdot \mathbf{a}_{4} \mathbf{f} \cdot \mathbf{a}_{5}\left[\mathbf{f a}_{1} \mathbf{a}_{2} \mathbf{a}_{3}\right]-\mathbf{f} \cdot \mathbf{a}_{2} \mathbf{f} \cdot \mathbf{a}_{3}\left[\mathbf{f a}_{1} \mathbf{a}_{4} \mathbf{a}_{5}\right]} \\
& +\mathbf{f} \cdot \mathbf{a}_{2} \mathbf{f} \cdot \mathbf{a}_{4}\left[\mathbf{f} \mathbf{a}_{1} \mathbf{a}_{3} \mathbf{a}_{5}\right] \text {. }
\end{aligned}
$$

It is multilinear with respect to $\mathbf{a}_{1}, \mathbf{a}_{2}, \mathbf{a}_{3}, \mathbf{a}_{4}, \mathbf{a}_{5}$ and satisfies

$$
\begin{aligned}
{\left[\mathbf{a}_{1} ; \mathbf{a}_{2} \mathbf{a}_{3} ; \mathbf{a}_{4} \mathbf{a}_{5}\right] } & =-\left[\mathbf{a}_{1} ; \mathbf{a}_{4} \mathbf{a}_{5} ; \mathbf{a}_{2} \mathbf{a}_{3}\right]=\left[\mathbf{a}_{2} ; \mathbf{a}_{3} \mathbf{a}_{5} ; \mathbf{a}_{1} \mathbf{a}_{4}\right] \\
=\left[\mathbf{a}_{3} ; \mathbf{a}_{5} \mathbf{a}_{4} ; \mathbf{a}_{2} \mathbf{a}_{1}\right] & =\left[\mathbf{a}_{4} ; \mathbf{a}_{1} \mathbf{a}_{2} ; \mathbf{a}_{5} \mathbf{a}_{3}\right]=\left[\mathbf{a}_{5} ; \mathbf{a}_{4} \mathbf{a}_{1} ; \mathbf{a}_{3} \mathbf{a}_{2}\right] .
\end{aligned}
$$

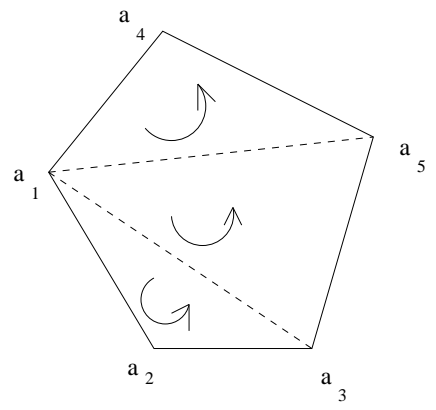

Fig. 4. Interpretation of $\left[\mathbf{a}_{1} ; \mathbf{a}_{2} \mathbf{a}_{3} ; \mathbf{a}_{5} \mathbf{a}_{4}\right]$. 


\section{Theorem proving with Clifford bracket algebra}

For geometric theorems of linear constructive type, i.e., the coordinates of newly constructed points can be explicitly expressed by those of previously constructed ones, Clifford bracket algebra can provide amazing simplification in eliminating points sequentially by shortening expressions, and by producing more factors to make the expressions more decomposed.

We present below two examples. According to X.-S. Gao, they are the only two theorems whose proofs are theoretically easy by any current algebraic method but whose computing cannot be finished by any kind of software and hardware currently available in research institutes.

Example 1. (Miquel Theorem) Four circles intersect pairwise at pairs of points $(1, a)$, $(2, c),(3, b),(4, d)$ respectively. If $1,2,3,4$ are cyclic, so are $a, b, c, d$.

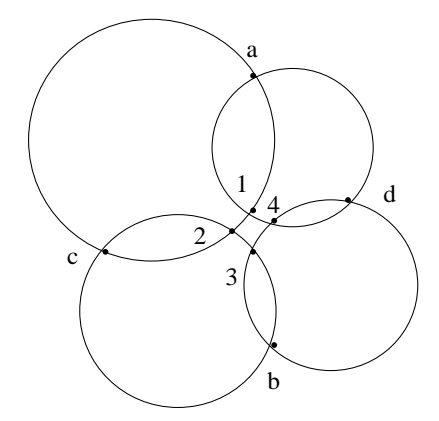

Fig. 5. Miquel Theorem.

The following is a constructive sequence for this theorem: $a, b, 1,2,3,4$ are free points, $c=12 a \cap 23 b$, and $d=14 a \cap 34 b$.

We need to prove that $[\mathbf{1 2 3 4}]$ is a factor of $[\mathbf{a b c d}]$. By eliminating $\mathbf{c}, \mathbf{d}$ from $[\mathbf{a b c d}]$ using

$$
\begin{aligned}
& \mathbf{c}=1 \cdot \mathbf{a}[23 \mathrm{~b} 1][23 \mathrm{ba}] 2-[23 \mathrm{ba}][3 \mathrm{~b} ; 1 \mathrm{a}]_{2} \mathbf{1}+[23 \mathrm{~b} 1][3 \mathrm{~b} ; 1 \mathrm{a}]_{2} \mathrm{a} \\
& \mathbf{d}=1 \cdot \mathbf{a}[43 \mathrm{~b} 1][43 \mathrm{ba}] 4-[43 \mathrm{ba}][3 \mathrm{~b} ; 1 \mathrm{a}]_{4} 1+[43 \mathrm{~b} 1][3 \mathrm{~b} ; 1 \mathrm{a}]_{4} \mathrm{a}
\end{aligned}
$$

we get

$$
\begin{aligned}
& {[\mathrm{abcd}]=(\underline{\mathbf{1} \cdot \mathbf{a}})^{2}[\mathbf{a b 2 4}][23 \mathrm{~b} 1][23 \mathrm{ba}][43 \mathrm{~b} 1][43 \mathrm{ba}]} \\
& -1 \cdot \mathrm{a}[\mathrm{ab21}][23 \mathrm{~b} 1][23 \mathrm{ba}][43 \mathrm{ba}][3 \mathrm{~b} ; 1 \mathrm{aa}]_{4} \\
& +1 \cdot \mathrm{a}[\mathrm{ab} 41][43 \mathrm{b1}][23 \mathrm{ba}][43 \mathrm{ba}][3 \mathrm{~b} ; 1 \mathrm{a}]_{2} \\
& =\mathbf{a} \cdot \mathbf{b}[123 \mathrm{~b}][134 \mathrm{~b}][124 \mathrm{a}]+\mathbf{1} \cdot 4[123 \mathrm{~b}][12 \mathrm{ab}][34 \mathrm{ab}] \\
& =3 \cdot \mathrm{b}[12 \mathrm{ab}][14 \mathrm{ab}][1234] \text {. }
\end{aligned}
$$

The underlined expressions in the first step are generically nonzero factors of [abcd], and are removed in the subsequent step. The last step can be finished with Maple V.5 installed on a Pentium III/500MHz IBM compatible PC, and takes 0.045 seconds.

The nondegeneracy condition of the proof is that $12 a, 23 b, 34 b, 41 a$ must be triplets of pairwise different generalized points, and 123ab, 134ab are quintuplets of non-cyclic generalized points. 
We can go one step further to get a beautiful formula by normalizing $\mathbf{c}, \mathbf{d}$ in (3.10):

$$
\begin{aligned}
& \mathbf{c} \cdot \mathbf{a}=\mathbf{1} \cdot \mathbf{2 1} \cdot \mathbf{a}[23 \mathrm{ab}]^{2}, \\
& \mathbf{d} \cdot \mathbf{b}=\mathbf{3} \cdot 4 \mathbf{3} \cdot \mathbf{b}[41 \mathrm{ab}]^{2} .
\end{aligned}
$$

Theorem. For 6 generic generalized points $a, b, 1,2,3,4$ in the plane, let $c=12 a \cap 23 b, d=$ $14 a \cap 34 b$, then

$$
\frac{[\mathrm{abcd}]}{\mathrm{a} \cdot \mathrm{cb} \cdot \mathrm{d}}=\frac{[1234]}{1 \cdot 23 \cdot 4} \frac{[12 \mathrm{ab}][34 \mathrm{ab}]}{[23 \mathrm{ab}][41 \mathrm{ab}]} \text {. }
$$

Example 2. The five-circle theorem in the beginning of this chapter. We need to prove $[$ abcd $]=0$.

First we compute $a=A B 1 \cap A E 5$ and eliminate constrained points from the brackets:

$$
\begin{aligned}
& \mathbf{a}=\mathbf{B} \cdot \mathbf{1}[\text { AE5B }][\text { AE51 }] \text { A }+[\text { AE51 }][\text { B1; E5 }]_{\mathbf{A}} \mathrm{B} \\
& -[\mathrm{AE} 5 \mathrm{~B}][\mathrm{B} 1 ; \mathbf{E} 5]_{\mathrm{A}} \mathbf{1} \\
& =\mathbf{f} \cdot \mathbf{C B} \cdot \mathbf{C}[\mathrm{fABE}][\mathrm{fBCE}][\mathrm{fBDE}][\mathrm{AB} ; \mathrm{DE}] \mathbf{A} \\
& +\mathbf{A} \cdot \mathbf{E}[\mathbf{f A B C}][\mathrm{fBCE}][\mathrm{AB} ; \mathrm{DE}][\mathrm{BC} ; \mathrm{DE}] \mathbf{B} \\
& -\mathbf{A} \cdot \mathbf{B}[\mathrm{fBDE}][\mathrm{BC} ; \mathbf{D E}] \mathbf{1} \text {. }
\end{aligned}
$$

By symmetry, $\mathbf{b}$ can be derived from a by interchanging $\mathbf{A} \leftrightarrow \mathbf{B}, \mathbf{C} \leftrightarrow \mathbf{E}$ :

$$
\begin{aligned}
& \mathbf{b}=\mathbf{B} \cdot \mathbf{C}[\mathbf{f A B E}][\mathbf{f A C E}][\mathrm{AB} ; \mathrm{CD}][\mathrm{EA} ; \mathrm{CD}] \mathrm{A} \\
& -\mathbf{f} \cdot \mathbf{E} \mathbf{A} \cdot \mathbf{E}[\mathbf{f A B C}][\mathbf{f A C D}][\mathbf{f A C E}][\mathrm{AB} ; \mathrm{CD}] \mathbf{B} \\
& +\mathbf{A} \cdot \mathbf{B}[\mathbf{f A C D}][\mathbf{E A} ; \mathbf{C D}] \mathbf{1} .
\end{aligned}
$$

So

$$
\begin{array}{r}
\mathbf{a} \wedge \mathbf{b}=-\mathbf{A} \cdot \mathbf{E B} \cdot \mathbf{C}[\mathbf{f A C E}][\mathbf{f B C E}][\mathbf{A B} ; \mathbf{C D}][\mathbf{A B} ; \mathbf{D E}] \mathbf{A} \wedge \mathbf{B} \\
+\mathbf{A} \cdot \mathbf{B} \mathbf{B} \cdot \mathbf{C}[\mathbf{f B D E}][\mathbf{E A} ; \mathbf{C D}] \mathbf{A} \wedge \mathbf{1} \\
+\mathbf{A} \cdot \mathbf{B A} \cdot \mathbf{E}[\mathbf{f A C D}][\mathbf{B C} ; \mathbf{D E}] \mathbf{B} \wedge \mathbf{1}
\end{array}
$$

By symmetry, $\mathbf{c} \wedge \mathbf{d}$ can be obtained from $\mathbf{a} \wedge \mathbf{b}$ by interchanging $\mathbf{a} \leftrightarrow \mathbf{d}, \mathbf{b} \leftrightarrow \mathbf{c}, \mathbf{A} \leftrightarrow$ $\mathrm{D}, \mathrm{B} \leftrightarrow \mathrm{C}, \mathbf{1} \leftrightarrow \mathbf{3}, \mathbf{4} \leftrightarrow \mathbf{5}$. Then

$$
\begin{aligned}
& {[\mathbf{a b c d}]=\mathbf{A} \cdot \mathbf{E} \mathbf{C} \cdot \mathbf{D}[\mathbf{A B} ; \mathbf{C D}][\mathbf{A B} ; \mathbf{D E}][\mathbf{f A B C}]} \\
& {[\mathbf{f A B D}][\mathbf{f A C E}][\mathbf{f B C D}] \mathbf{f} \cdot \mathbf{E} \mathbf{D} \cdot \mathbf{E}} \\
& -\mathbf{A} \cdot \mathbf{E} \mathbf{C} \cdot \mathbf{D}[\mathbf{A B} ; \mathbf{D E}][\mathbf{B C} ; \mathbf{D E}][\mathbf{f A B D}] \\
& {[\mathbf{f A C E}][\mathbf{f B C D}][\mathbf{f C D E}] \mathbf{f} \cdot \mathbf{A ~ A} \cdot \mathbf{B}} \\
& +\mathbf{A} \cdot \mathbf{E} \mathbf{C} \cdot \mathbf{D}[\mathbf{A B} ; \mathbf{D E}]^{2}[\mathbf{f A B D}][\mathbf{f A C E}] \\
& ([\mathrm{BC} ; \mathbf{D E}][\mathrm{fABC}] \mathbf{C} \cdot \mathbf{D}-[\mathbf{A B} ; \mathbf{C D}][\mathrm{fCDE}] \mathbf{B} \cdot \mathbf{C}) \\
& -\mathbf{A} \cdot \mathbf{B} \mathbf{D} \cdot \mathbf{E}[\mathrm{AB} ; \mathrm{CD}][\mathrm{EA} ; \mathrm{CD}][\mathrm{fABC}] \\
& {[\mathbf{f A C D}][\mathbf{f B C D}][\mathrm{fBDE}] \mathbf{f} \cdot \mathbf{E} \mathbf{A} \cdot \mathbf{E}} \\
& +\mathbf{A} \cdot \mathbf{B} \mathbf{D} \cdot \mathbf{E}[\mathbf{E A} ; \mathbf{B C}][\mathbf{E A} ; \mathbf{C D}][\mathbf{f A B C}] \\
& {[\mathbf{f A B E}][\mathbf{f A C D}][\mathbf{f B D E}] \mathbf{f} \cdot \mathbf{D} \mathbf{C} \cdot \mathbf{D}} \\
& +\mathbf{A} \cdot \mathbf{B} \mathbf{D} \cdot \mathbf{E}[\mathbf{E A} ; \mathbf{C D}]^{2}[\mathrm{fACD}][\mathrm{fBDE}] \\
& ([\mathrm{AB} ; \mathrm{CD}][\mathbf{f A B E}] \mathbf{B} \cdot \mathbf{C}-[\mathbf{E A} ; \mathbf{B C}][\mathbf{f B C D}] \mathbf{A} \cdot \mathbf{B}) \\
& =0 \text {. }
\end{aligned}
$$


The last step takes 0.315 seconds to finish on our computer. All together 45 factors are removed in the whole computing procedure:

\section{$[\mathbf{f A B C}]^{7}[\mathbf{f B C D}]^{7}[\mathbf{f A B E}]^{6}[\mathbf{f C D E}]^{6}[\mathbf{f A D E}]^{4}(\mathbf{B} \cdot \mathbf{C})^{4}[\mathbf{A} ; \mathbf{C E} ; \mathbf{D B}]^{2}$ $(\mathbf{A} \cdot \mathbf{B})^{2}(\mathbf{C} \cdot \mathbf{D})^{2}[\mathbf{f A C E}][\mathbf{f B C E}][\mathbf{f B D E}] \mathbf{A} \cdot \mathbf{E} \mathbf{D} \cdot \mathbf{E}$.}

The nondegeneracy condition of the proof is that $A B C, B C D, C D E, D E A, E A B$ are triplets of non-collinear points.

It must be pointed out that both theorems can be elegantly proved in a completely coordinate-free manner in a few lines with our pure algebraic method. The results will be presented in another paper, where several new bracket expressions are constructed and the corresponding groups of relations from the generalized Grassmann-Plücker relations are established.

\section{References}

[1] S.-C. Chou (1988): Mechanical Geometry Theorem Proving, D. Reidel, Dordrecht, Boston.

[2] S.-C. Chou, X.-S. Gao and J.-Z. Zhang (1994): Machine Proofs in Geometry, World Scientific, Singapore, 1994.

[3] S. Fevre, D. Wang (1999): Combining Clifford Algebraic Computing and Term-Rewriting for Geometric Theorem Proving. Fundamenta Informaticae 39 (1-2): 85-104.

[4] T. Havel (1991): Some examples of the use of distances as coordinates for Euclidean geometry, J. Symbolic Computat. 11: 579-593.

[5] T. Havel (1995): Geometric algebra and Möbius sphere geometry as a basis for Euclidean invariant theory, in Invariant Methods in Discrete and Computational Geometry, N. L. White (ed.), pp. 245-256, D. Reidel, Dordrecht.

[6] D. Hestenes, G. Sobczyk (1984): Clifford Algebra to Geometric Calculus, D. Reidel, Dordrecht, Boston.

[7] D. Hestenes (1991): The design of linear algebra and geometry, Acta Appl. Math. 23: 65-93.

[8] X.-R. Hou, H. Li, D. Yang and L. Yang (2001): "Russian killer" No. 2: a challenging geometric theorem with machine vs. human proofs. Math. Intelligencer 23(1): 1-7.

[9] H. Li (1994): New explorations of automated theorem proving in geometries. Doctoral Dissertation, Peking University, Beijing.

[10] H. Li, M.-t. Cheng (1997): Proving theorems in elementary geometry with Clifford algebraic method. Chinese Advances in Math. 26(4): 357-371.

[11] H. Li (1997): On mechanical theorem proving in differential geometry - local theory of surfaces. Science in China, Ser. A, 40(4): 350-356.

[12] H. Li, H. Shi (1997): On Erdos' ten-point problem. Acta Math. Sinica, New Series 13(2): 221230.

[13] H. Li, M.-t. Cheng (1998): Clifford algebraic reduction method for mechanical theorem proving in differential geometry. Journal of Automated Reasoning 21: 1-21.

[14] H. Li (1998): Some applications of Clifford algebra to geometries. Automated Deduction in Geometries, LNAI 1669, X.-S. Gao, D. Wang, L. Yang (eds.), pp. 156-179.

[15] H. Li (2000): Vectorial equation-solving for mechanical geometry theorem proving. Journal of Automated Reasoning 25: 83-121. 
[16] H. Li (2000): Doing geometric research with Clifford algebra. Clifford Algebras and Their Applications in Mathematical Physics, Volume 1: Algebra and Physics, R. Ablamowicz and B. Fauser (eds.), Birkhauser Boston, pp. 195-218.

[17] H. Li (2000): Mechanical theorem proving in differential geometry, Mathematics Mechanization and Applications, X.-S. Gao and D. Wang (eds.), Academic Press, London, pp. 147-174.

[18] H. Li (2000): Clifford algebra approaches to automated geometry theorem proving. Mathematics Mechanization and Applications, X.-S. Gao and D. Wang (eds.), Academic Press, London, pp. 205-230.

[19] H. Li, D. Hestenes and A. Rockwood (2000): Generalized homogeneous coordinates for computational geometry, in Geometric Computing with Clifford Algebra, G. Sommer (ed.), Springer Heildeberg, pp. 27-60.

[20] B. Mourrain, N. Stolfi (1995): Computational symbolic geometry, in Invariant Methods in Discrete and Computational Geometry, N. L. White (ed.), pp. 107-139, D. Reidel, Dordrecht, Boston.

[21] H. Crapo, J. Richter-Gebert (1994): Automatic proving of geometric theorems, in: Invariant Methods in Discrete and Computational Geometry, N. White (ed.), pp. 107-139, D. Reidel, Dordrecht, Boston.

[22] J. Richter-Gebert (1995): Mechanical theorem proving in projective geometry, Annals of Math. and Artificial Intelligence 13: 159-171.

[23] J. Seidel (1952): Distance-geometric development of two-dimensional Euclidean, hyperbolic and spherical geometry I, II, Simon Stevin 29: 32-50, 65-76.

[24] B. Sturmfels (1993): Algorithms in Invariant Theory, Springer Wien, New York.

[25] B. Sturmfels, N. White (1989): Gröbner bases and invariant theory, Adv. Math. 76: 245-259.

[26] N. White (1991): Multilinear Cayley factorization, J. Symbolic Computat. 11: 421-438.

[27] W. Whiteley (1991): Invariant computations for analytic projective geometry, J. Symbolic Computat. 11: 549-578.

[28] W.-t. Wu (1994): Mechanical Theorem Proving in Geometries: Basic Principle (translated from Chinese edition 1984), Springer Wien.

[29] W.-t. Wu (2000): Mathematics Mechanization. Science Press \& Kluwer Academic, Beijing \& Dordrecht. 